\title{
Collagen fiber orientation disorder from H\&E images is prognostic for early stage breast cancer: clinical trial validation
}

Haojia Li $\mathbb{D}^{1 凶}$, Kaustav Bera (D) ${ }^{1}$, Paula Toro ${ }^{1}$, PingFu Fu ${ }^{2}{ }^{2}$, Zelin Zhang ${ }^{3}$, Cheng Lu', Michael Feldman ${ }^{4}$, Shridar Ganesan $^{5}$, Lori J. Goldstein ${ }^{6}$, Nancy E. Davidson ${ }^{7}$, Akisha Glasgow ${ }^{8}$, Aparna Harbhajanka ${ }^{8}$, Hannah Gilmore ${ }^{8}$ and Anant Madabhushi iD $^{1,9 凶}$

Collagen fiber organization has been found to be implicated in breast cancer prognosis. In this study, we evaluated whether computerized features of Collagen Fiber Orientation Disorder in Tumor-associated Stroma (CFOD-TS) on Hematoxylin \& Eosin (H\&E) slide images were prognostic of Disease Free Survival (DFS) in early stage Estrogen Receptor Positive (ER+) Invasive Breast Cancers (IBC). A Cox regression model named $M_{C F O D-T S}$, was constructed using cohort $S_{t}(N=78)$ to predict DFS based on CFOD-TS features. The prognostic performance of $M_{C F O D-T S}$ was validated on cohort $S_{v}(N=219)$, a prospective clinical trial dataset (ECOG 2197). $M_{\text {CFOD-TS }}$ was prognostic of DFS in both $S_{t}$ and $S_{v}$, independent of clinicopathological variables. Additionally, the molecular pathways regarding cell cycle regulation were identified as being significantly associated with $\mathrm{M}_{\mathrm{CFOD}-\mathrm{TS}}$ derived risk scores. Our results also found that collagen fiber organization was more ordered in patients with short DFS. Our study provided a H\&E imagebased pipeline to derive a potential prognostic biomarker for early stage ER+ IBC without the need of special collagen staining or advanced microscopy techniques.

npj Breast Cancer (2021)7:104; https://doi.org/10.1038/s41523-021-00310-z

\section{INTRODUCTION}

Breast cancer is the second leading cause of cancer death among women in the United States, with approximately $80 \%$ of these cancers being Estrogen Receptor Positive (ER+) and 64\% being early stage (Stage I \& II in Tumor, Node, Metastasis staging system) ${ }^{1}$. The standard treatment regimen for early stage ER+ Invasive Breast Cancer (IBC) patients is breast conserving surgery followed by hormonal therapy. While chemotherapy is able to significantly reduce the breast cancer associated mortality rate ${ }^{2}$, most early stage ER+ IBC patients do not receive added benefit from adjuvant chemotherapy ${ }^{3}$. Oncotype $d x(\mathrm{Odx})^{4}$ is a 21-gene expression assay to assess the likelihood of cancer recurrence and the need for adjuvant chemotherapy for early stage $\mathrm{IBC}^{5}$. High Odx risk category indicates a high risk of recurrence and low Odx category suggests a low recurrence risk. The intermediate Odx category, however, has a more ambiguous prognostic/predictive meaning. While the Odx test is now routinely used in the clinical setting, Odx and other similar multi-gene assays tend to be timeconsuming, high-cost, tissue-destructive and are not widely available in many countries.

Numerous studies have demonstrated that the interaction between tumor cells and extracellular matrix (ECM) plays a critical role in breast cancer progression and metastasis ${ }^{6-9}$. Collagen is the most abundant ECM component in breast cancer, which provides tensile strength and structural support to the tumor tissues ${ }^{10}$. Collagen fiber organization has been shown to be different between IBC and benign breast tumor, as well as between more and less aggressive IBC in both in-vivo/vitro models ${ }^{7,11-14}$. Using Second Harmonic Generation (SHG) based microscopy, Golaraei et al. $^{15}$ observed an ultrastructure alteration in collagen fiber architecture in breast cancers, as compared to normal breast tissue. Friedl et al. ${ }^{16}$ observed that the focal sites of breast cancer cell micro-invasion often co-existed with stiff aligned collagen fibers. In addition, some studies ${ }^{7,11,17}$ have shown that organized collagen architecture in breast cancer was associated with a worse prognosis, as evidenced by SHG and Laser Scanning Microscopy (LSM) techniques.

Taken together, these studies strongly suggest that collagen fiber organization is associated with prognosis of breast cancer. However, the reported studies have employed advanced imaging techniques such as SHG or LSM microscopy ${ }^{11,17-19}$, techniques not routinely used in pathology labs or in clinical practice.

With the advent of digital pathology 20 , there has been substantial interest in exploring the role of computationally extracted quantitative histomorphometric attributes based on Hematoxylin \& Eosin (H\&E) stained Whole Slide Image (WSI) in diagnosis and prognosis across multiple cancer types ${ }^{21-25}$. In the context of breast cancer, histomorphometric features relating to measurements of shape, texture, and orientation of individual cancer nuclei both from the tumor epithelium ${ }^{21-23}$ and tumorassociated stroma have been implicated in cancer progression and patient survival ${ }^{26}$.

We present a digital pathology-based pipeline that is able to prognosticate DFS in early stage ER+ IBC by automatically quantifying the Collagen Fiber Orientation Disorder in Tumorassociated Stroma (CFOD-TS) directly from digitized routine H\&E image. Our computational pathology-derived biomarker could be more readily transitioned into clinical practice by taking advantage of the rapid increase in the availability of WSI scanning ${ }^{27}$, compared to the SHG/LSM microscopy-based biomarkers.

\footnotetext{
${ }^{1}$ Case Western Reserve University, Department of Biomedical Engineering, Cleveland, OH, USA. ${ }^{2}$ Case Western Reserve University, Department of Population and Quantitative Health Sciences, School of Medicine, Cleveland, OH, USA. ${ }^{3}$ Nanjing University of Information Science and Technology, Jiangsu Key Laboratory of Big Data Analysis Technique, Nanjing, China. ${ }^{4}$ University of Pennsylvania Perelman School of Medicine, Philadelphia, PA, USA. ${ }^{5}$ Rutgers Cancer Institute of New Jersey, New Brunswick, NJ, USA. ${ }^{6}$ Fox Chase Cancer Center, Philadelphia, PA, USA. ${ }^{7}$ Fred Hutchinson Cancer Research Center, University of Washington, and Seattle Cancer Care Alliance, Seattle, WA, USA. ${ }^{8}$ University

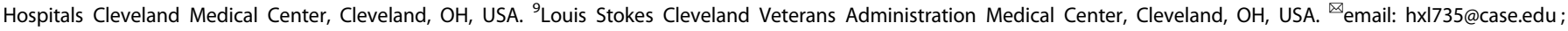
axm788@case.edu
} 
Specifically, the automated pipeline consists of image processing techniques combined with a machine learning model to segment tumor-associated stroma on H\&E slide images, from which we subsequently detected the collagen fiber orientations. Subsequently, we utilized entropy theory ${ }^{28}$ to calculate CFOD-TS, in order to quantitatively characterize the degree of disorder of the collagen fiber orientations at the tumor leading edge as well as across the entire tumor region. Following that, 78 patients (number of DFS event $=34$ ) with early stage ER+ IBC identified from The Cancer Genome Atlas Breast Invasive Carcinoma (TCGA $B R C A$ ) were used as the training set $\left(S_{t}\right)$ to construct a Cox proportional regression model (MCFOD-TS) to predict DFS based on the CFOD-TS features. 219 breast cancer patients (number of DFS event $=64$ ) from the completed Eastern Cooperative Oncology Group 2197 (ECOG 2197) were employed $\left(S_{v}\right)$ for validating $M_{C F O D-}$ Ts. ECOG $2197^{29}$ is a randomized phase III clinical trial to compare the effectiveness of two combination chemotherapy regimens in treating early stage IBC (no significant difference observed in cancer outcome between the two regimens) ${ }^{29}$. The prognostic significance of $M_{C F O D-T S}$ was also validated on intermediate (indeterminate) Odx category as well as the subgroup of lymph node negative $(\mathrm{LN}-)$ and lymph node positive $(\mathrm{LN}+)$ patients in $\mathrm{S}_{\mathrm{v}}$. Additionally, we explored the relationship between CFOD-TS characterized phenotype and the underlying genotype utilizing the genomic data in $\mathrm{S}_{\mathrm{t}}$.

\section{RESULTS}

\section{Patient characteristics}

Clinicopathological variables for $\mathrm{S}_{\mathrm{t}}$ and $\mathrm{S}_{\mathrm{v}}$ are provided in Table 1. In $S_{t}, 34$ patients (45\%) had an event (recurrence or death) with median DFS being 1379 days versus 65 patients (30\%) with an event and median DFS of 3517 days in $\mathrm{S}_{\mathrm{v}}$. All patients in $\mathrm{S}_{\mathrm{v}}$ received adjuvant chemotherapy while treatment related information was only available for 6 patients in $S_{t}$, with 4 of them receiving adjuvant chemotherapy. Other than tumor size and age, no clinicopathological variables were found to be significantly different between $S_{t}$ and $S_{v}$. $S_{v}$ was considered a rigorous validation set of $M_{C F O D-T S}$ given that the relative homogeneity in terms of treatment regimen (all patients received chemotherapy) and cancer clinical characteristics enabled the outcome to be more reflective of the inherent aggressiveness of breast cancer.

\section{Experiment 1: CFOD-TS prognostic of DFS in early stage ER+ breast cancer}

A total of 8 CFOD-TS features were identified in the LASSO regularized Cox regression modeling with DFS on $S_{t}(N=78)$ and integrated into $M_{\text {CFOD-TS. The number of top features was }}$ determined as approximately $10 \%$ of the patient number in $\mathrm{S}_{\mathrm{t}}$. All 8 CFOD-TS features were found to be significantly $(p<0.05$ from WRST) lower in patients with short DFS (with an event and DFS $<5$ years) compared to those with long DFS (DFS $>=5$ years) in $S_{t+v}\left(S_{t}+S_{v}\right)$. The eight identified features consisted of the following: CFOD-TS in the FOV of 50, 100, $250 \mu \mathrm{m}$ at the tumor leading edge and CFOD-TS in FOV of 75, 125, 150, 200, $225 \mu \mathrm{m}$ across the whole tumor region. Notably, among the eight identified top features, CFOD-TS features derived from relatively small FOVs were found to be more distinguishable (smaller $p$ value from WRST) between short and long DFS patient groups (Fig. 1).

Figure 2 shows the visualization of collagen fiber organization at the tumor edge for a short and long DFS patient respectively. An aligned collagen fiber organization could be observed in the highrisk patient based on both feature map (Fig. 2c, g) and closer visual inspection of the cancer tissues (Fig. 2d, h).

A risk category $R_{M}$ was predicted from $M_{C F O D-T S}$ by dichotomizing the sum of a linear combination of the identified eight top features as described in the "Statistical analysis" section. The KM survival
Table 1. Summary of clinicopathological variables and Odx risk categories in $\mathrm{S}_{\mathrm{t}}$ and $\mathrm{S}_{\mathrm{v}}$.

\begin{tabular}{|c|c|c|}
\hline Clinical variable & $\mathrm{S}_{\mathrm{t}}: N(\%)$ & $\mathrm{S}_{\mathrm{v}}: N(\%)$ \\
\hline \multicolumn{3}{|l|}{ Lymph node } \\
\hline node-- & $23(29 \%)$ & 116 (53\%) \\
\hline node + & $36(46 \%)$ & $103(47 \%)$ \\
\hline unknown & 19 (25\%) & $0(0 \%)$ \\
\hline \multicolumn{3}{|l|}{$P R$} \\
\hline $\mathrm{pr}+$ & 66 (85\%) & 185 (84\%) \\
\hline $\mathrm{pr}-$ & 12 (15\%) & $34(16 \%)$ \\
\hline \multicolumn{3}{|l|}{ HER2 } \\
\hline her2+ & 15 (19\%) & $40(18 \%)$ \\
\hline her2- & $33(42 \%)$ & $88(40 \%)$ \\
\hline unknown & 30 (39\%) & 91 (42\%) \\
\hline Tumor grade & Not available & \\
\hline grade 1 & & $32(15 \%)$ \\
\hline grade 2 & & 107 (49\%) \\
\hline grade 3 & & $56(26 \%)$ \\
\hline unknown & & $24(10 \%)$ \\
\hline \multicolumn{3}{|l|}{ Tumor size } \\
\hline$\leq 2 \mathrm{~cm}$ & $20(26 \%)$ & 95 (43\%) \\
\hline$>2 \mathrm{~cm}$ & 58 (74\%) & $122(56 \%)$ \\
\hline unknown & $0(0 \%)$ & $2(1 \%)$ \\
\hline \multicolumn{3}{|l|}{ Age } \\
\hline$\geq 50$ years & $21(27 \%)$ & 89 (41\%) \\
\hline$<50$ years & $56(72 \%)$ & 130 (59\%) \\
\hline unknown & $1(1 \%)$ & $0(0 \%)$ \\
\hline Oncotype Dx & Not available & \\
\hline Low & & $14(6 \%)$ \\
\hline Intermediate & & $28(13 \%)$ \\
\hline High & & 17 (8\%) \\
\hline Unknown & & 160 (73\%) \\
\hline \multicolumn{3}{|l|}{ Race } \\
\hline Caucasian & $61(78 \%)$ & 198 (90\%) \\
\hline Non-Caucasian & 12 (15\%) & $21(10 \%)$ \\
\hline Unknown & $5(7 \%)$ & $0(0 \%)$ \\
\hline Histology type & & Not available \\
\hline Ductal invasive carcinoma & $60(77 \%)$ & \\
\hline Lobular invasive carcinoma & $11(14 \%)$ & \\
\hline Unknown & $7(9 \%)$ & \\
\hline
\end{tabular}

curves of DFS between $R_{M}{ }^{H}$ and $R_{M}{ }^{L}$ groups are plotted for both $S_{t}$ (Fig. 3a) and $S_{v}$ (Fig. 3b). A significantly favorable outcome in $R_{M}{ }^{L}$ compared to $\mathrm{R}_{M}{ }^{H}$ was observed with $\mathrm{HR}=3.2(95 \% \mathrm{Cl}=1.12-9.17$, $p=0.00086)$ on $\mathrm{S}_{\mathrm{t}}$ and $\mathrm{HR}=2.64(95 \% \mathrm{Cl}=1.39-5.03, p=0.000126)$ on $S_{v}$ using a log-rank test. In the subgroup survival analysis on $S_{v}$ (Fig. 3c, d), M MFOD-TS was also found to be prognostic in the subgroup of the $\mathrm{LN}-$ patients with $\mathrm{HR}=2.55(95 \% \mathrm{Cl}=1.09-5.98$, $p=0.031$ ) (Fig. $3 c)$ and $\mathrm{LN}+$ patients with $\mathrm{HR}=2.91 \quad(95 \%$ $\mathrm{Cl}=1.24-6.83, p=0.000728$ ) (Fig. 3d). Furthermore, $\mathrm{M}_{\text {CFOD-TS }}$ was found to significantly risk stratify intermediate Odx risk category patients ( $\mathrm{HR}=3.29,95 \% \mathrm{Cl}=1.13-9.54, p=0.0287$ ) (Fig. 3e).

\section{Experiment 2: Univariate and multivariate analysis}

Table 2 illustrates the results of univariate and multivariate analysis of clinicopathological variables/Odx risk category/ $R_{M}$ for DFS. In the univariate analysis setting, other than lymph node 
CFOD-TS in FOV of 50 um at the tumor leading edge

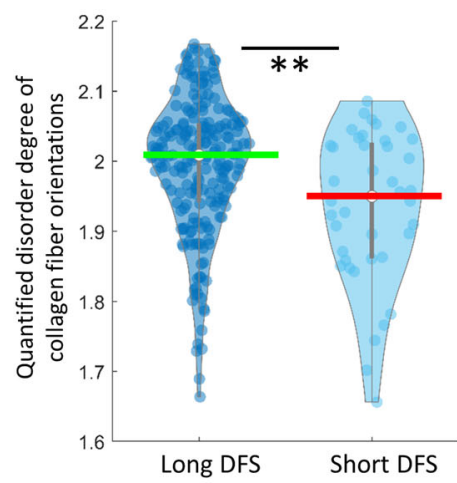

CFOD-TS in FOV of 75 um across the entire tumor

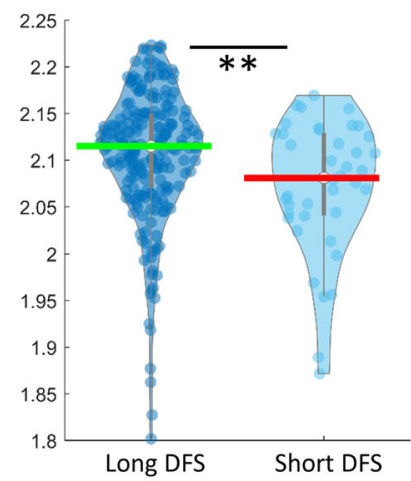

CFOD-TS in FOV of 100 um at the tumor leading edge

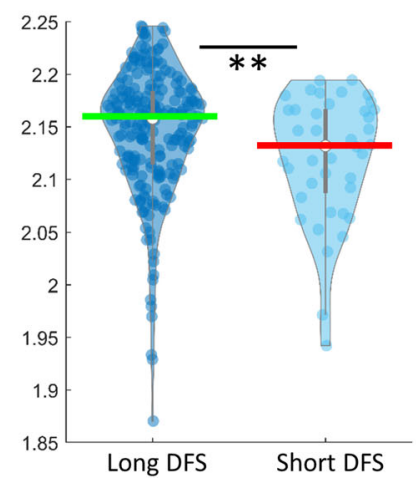

Fig. 1 Top CFOD-TS features distribution. Distribution of top three CFOD-TS features between patients with long DFS (DFS $\geq 5$ years) and short DFS (with an event \& DFS $<5$ years) in $S_{t+v}\left(S_{t}+S_{v}\right)$ with each point representing one single patient, including CFOD-TS features derived from FOV (left to right) in 50 um at tumor leading edge; in $75 \mu \mathrm{m}$ across the whole tumor and $100 \mu \mathrm{m}$ at the tumor leading edge.

status in $\mathrm{S}_{\mathrm{v}}$, neither clinicopathological variables nor Odx risk category was identified as being significantly prognostic of DFS on $\mathrm{S}_{\mathrm{t}}$ and $\mathrm{S}_{\mathrm{v}}$. Patients in $\mathrm{R}_{\mathrm{M}}{ }^{\mathrm{H}}$ had significantly worse DFS $(\mathrm{HR}=3.49$, $95 \% \mathrm{Cl}=1.6-7.6, p=0.001$ on $\mathrm{S}_{\mathrm{t}} ; \mathrm{HR}=2.67,95 \% \mathrm{Cl}=1.58-4.5$, $p=2.28 \mathrm{e}-04$ on $\mathrm{S}_{\mathrm{v}}$ ) compared to those in $\mathrm{R}_{\mathrm{M}}{ }^{\mathrm{L}}$. In the multivariate analysis controlling the effects of the clinicopathological variables, $R_{M}$ was found independently prognostic of DFS with $H R=3.45$ $(95 \% \mathrm{Cl}=1.03-12, p=0.04)$ on $\mathrm{S}_{\mathrm{t}}$ and $\mathrm{HR}=3.82(95 \% \mathrm{Cl}=$ $2.14-6.8, p=5.79 \mathrm{e}-06)$ on $\mathrm{S}_{\mathrm{v}}$. The prognostic significance of lymph node status in $S_{V}$ still held after adjusting the impact of other clinicopathological variables.

\section{Experiment 3: Identification of associated biological pathways with CFOD-TS features and enrichment analysis}

Out of the 60488 annotated genes for TCGA BRCA, 1062 genes (Supplementary List 1) were significantly differentially expressed between patients assigned to the $R_{M}{ }^{H}$ and $R_{M}{ }^{L}$. Among the $G O$ identified pathways with the 1062 genes as the input, ten molecular pathways (Supplementary Table 2) were considered as regulating the potential underpinning biological process of the CFOD-TS defined phenotype. Six of the pathways were directly related to extracellular or fiber organization while four pathways were chosen considering their biological significance in regulating cell development and cell division. None of the enrichment scores calculated by SSGSEA for the six pathways regarding ECM organization was significantly associated with $M_{\text {CFOD-TS }}$ derived risk scores. However, among the four identified pathways describing the cell development and division process, Cell Cycle Arrest (CCA) and Regulation of Cell Cycle (RCC) were identified to be significantly associated with $\mathrm{M}_{\text {CFOD-Ts }}$ risk scores, with the Pearson correlation coefficients respectively being -0.35 ( $p=$ $0.0017)$ and $-0.31(p=0.0069)$. CCA ${ }^{30}$ is a checkpoint in the cell cycle before proceeding to duplication and division. CCA was found to be overexpressed in $\mathrm{M}_{\mathrm{CFOD}-T \mathrm{~S}^{\mathrm{L}}}$ patients, suggesting that the mechanism facilitating DNA repair or cell cycle progression was differentially regulated in low risk compared to high risk patients. Similarly, RCC ${ }^{31}$ was also found to be negatively associated with $\mathrm{M}_{\mathrm{CFOD}-\mathrm{TS}}$ derived risk scores, suggesting a more dysregulated cell division process in $\mathrm{R}_{M}{ }^{\mathrm{H}}$ patients.

\section{DISCUSSION}

Increasing evidence has emerged to $6,7,11,17$ demonstrate the critical role of collagen fiber organization in breast cancer progression. While multiple studies ${ }^{11,17}$ have been conducted to identify prognostic biomarkers for invasive breast cancer (IBC) based on the quantitative measurement of collagen fiber organization, these studies have typically involved the use of advanced microscopy imaging technologies like Second Harmonic Generation (SHG) or Laser Scanning Microscopy (LSM) system. Those technologies, however, are far from translation into the routine clinical pathology workflow. With the advent of digital pathology, there has been substantial research interest in investigating computationally extracted quantitative histomorphometric attributes such as shape and spatial arrangement of cancer nuclei and immune cells on H\&E images for breast cancer prognosis $21,22,26,32$. However, to the best of our knowledge, no study has attempted to quantitatively measure collagen fiber organization directly on standard H\&E slide images and relate these measurements to breast cancer outcome.

The objective of this study is to quantitatively measure the organization of collagen fibers from routine $\mathrm{H} \& \mathrm{E}$ slide images alone and investigate its association with the outcome (DFS) for early stage $E R+I B C$. Utilizing a set of imaging processing techniques and a state-of-the-art deep learning model, we were able to quantitatively characterize Collagen Fiber Orientation Disorder in Tumor-associated Stroma (CFOD-TS) solely with routine $\mathrm{H} \& \mathrm{E}$ stained images, without the need of special collagen staining or advanced imaging and microscopy techniques. Following the CFOD-TS feature extraction, we constructed a Cox regression model ( $\mathrm{M}_{\mathrm{CFOD}-\mathrm{TS}}$ ) with CFOD-TS features to predict DFS, with a continuous risk score as the output. $\mathrm{M}_{\mathrm{CFOD}-\mathrm{TS}}$ was validated to be prognostic on an independent, archived clinical trial dataset (ECOG 2197) independent of clinicopathologic variables e.g. tumor grade, tumor size. $\mathrm{M}_{\mathrm{CFOD}-\mathrm{TS}}$ was also found to be prognostic on the patients in the subgroups of $\mathrm{LN}-, \mathrm{LN}+$ and intermediate Odx risk category. Additionally, the molecular pathways relevant to cell cycle regulation were identified as associated with the collagen fiber organization phenotype. We found the aligned and ordered organization of collagen fibers was indicative of a worse prognosis in terms of DFS for early stage ER+ IBC.

Oncotype $\mathrm{Dx}(\mathrm{Odx})$ is a multi-gene test which is prognostic and predictive of benefit of adjuvant chemotherapy for early stage $\mathrm{IBC}^{5}$. While the high and low Odx risk categories have very clear prognostic and predictive significance, the intermediate Odx risk category is more ambiguous and results in challenges to the physicians in interpreting results for patients assigned to this category, especially with making appropriate treatment decisions ${ }^{4}$. Our model was able to risk stratify patients in the intermediate Odx risk category with $\mathrm{HR}=3.29(95 \% \mathrm{Cl}=$ $1.13-9.54, p=0.0287)$. Most of the patients $(20 / 28)$ in intermediate Odx were identified as low risk by $M_{\text {CFOD-Ts }}$ and among the 8 patients in $\mathrm{R}_{\mathrm{M}}{ }^{\mathrm{H}}$ group, 7 patients recurred or died along the course of follow-up. While Odx test has been validated to be prognostic on ECOG $2197^{5}$, Odx test did not show prognostic significance in the univariate analysis for DFS (Table 2) in our study. Potential 
(a)
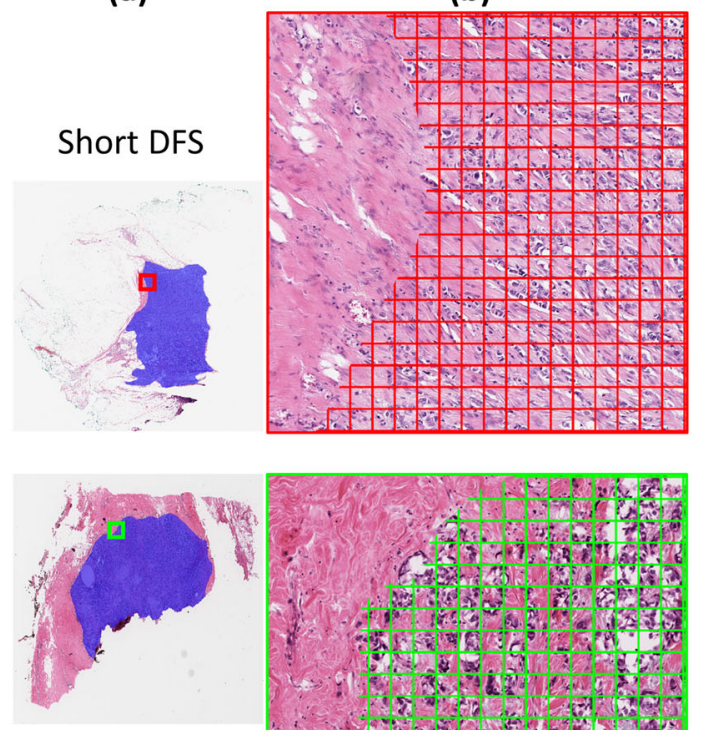

Long DFS

(e)

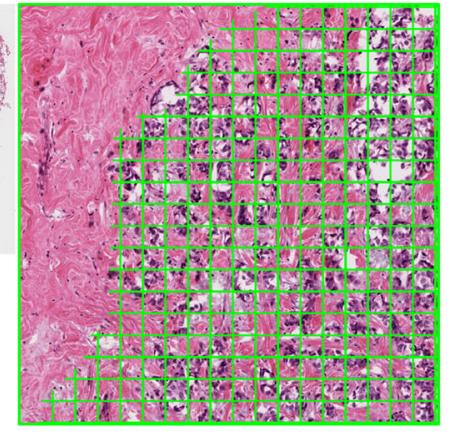

(f) (c)
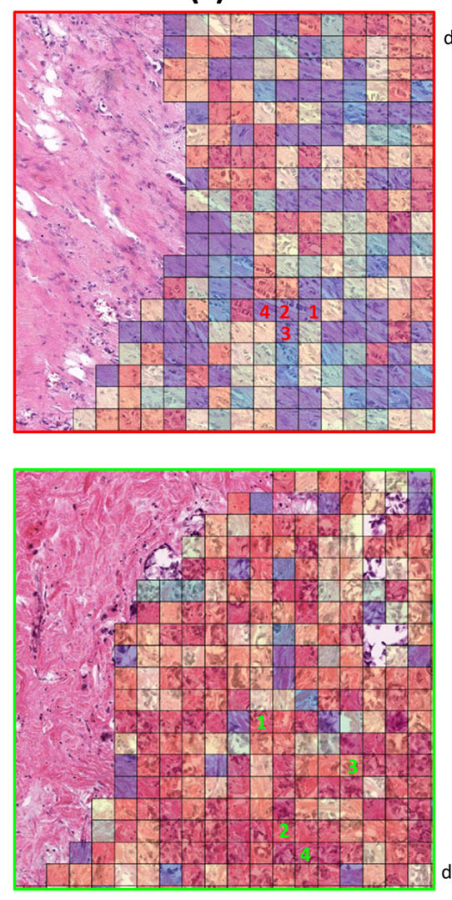

(g)

(d)

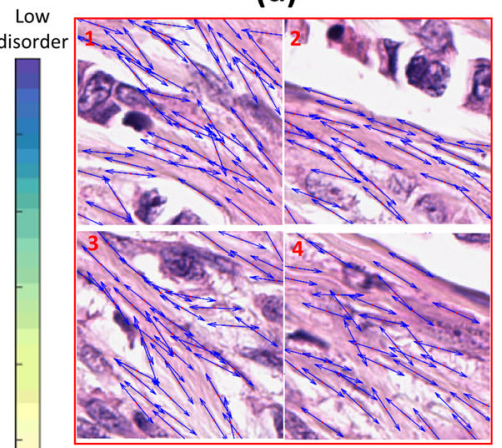

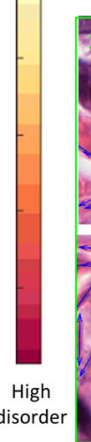

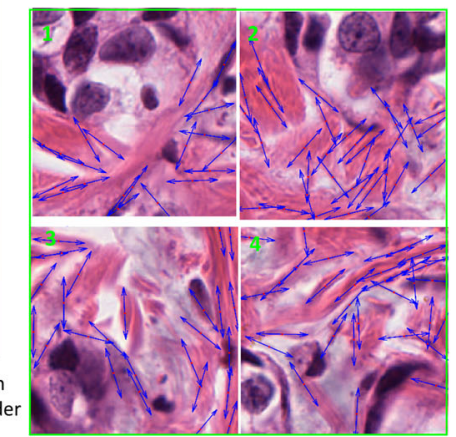

(h)

Fig. 2 Representative WSI of breast cancer tissue for patients with short DFS (top row) versus long DFS (bottom row). The first column (a, e) showed the original H\&E stained WSIs with tumor mask (blue) overlaid. The second column (b, e) showed the magnified tumor region at the leading edge, which was compartmentalized into a grid of smaller tumor neighborhoods of 50 um $\times 50$ um. The third column (c, $\mathbf{g}$ ) illustrates the quantified CFOD-TS in each individual tumor neighborhood within the tumor region in (b) and (f), where a warm color illustrates a higher feature value (higher disorder degree of fiber orientations); The fourth column (d, $\mathbf{h})$ illustrates the organization of the collagen fiber orientations (the orientations indicated by the blue arrows) in the tumor-associated stroma in representative tumor neighborhoods (position of each tumor neighborhood was indicated by the corresponding number on (c) and (g)). The patient with short DFS was characterized by a more aligned and ordered collagen fiber organization, while the patient with long DFS presented a higher degree of disorganization of collagen fiber orientation.

reasons for this could include (1) instead of using recurrence rate within five years, we used DFS as the end point, (2) we only had access to a subset of patients in ECOG 2197, and (3) we employed the updated risk thresholds as recommended by the TAILORx prospective study ${ }^{33}$ instead of the traditional categorization ${ }^{4}$ to divide the high/intermediate/low Odx category.

Quantitative measurement (Fig. 1) and qualitative visualization (Fig. 2) of the CFOD-TS features revealed the patients with short DFS tend to have a more aligned fiber organization, which appears to be in line with previous studies $8,12,34$. For example, multiple investigators ${ }^{15,16,35}$ have shown that in mice model, cancer cells preferentially invaded along aligned collagen fibers into the adjacent stroma, and focal sites of breast cancer cell micro-invasion often co-existed with stiff aligned collagen fibers. Also, Bredfeldt et $\mathrm{al}^{17}$. and Conklin et $\mathrm{al}^{11}$. found that the presence of straightened, aligned collagen fibers at a local tumor boundary observed on SHG images was indicator of poor prognosis for IBC patients. One of the advantages of our present pipeline is that we expanded the samples from tumor cores that previous studies ${ }^{11,17}$ have utilized in the collagen fiber organization analysis to WSIs, which harbor more comprehensive tumor characteristic information. A number of hypotheses ${ }^{14,34,36,37}$ have been proposed to demonstrate the mechanism behind how the aligned collagen fibers facilitated tumor migration and invasion. In the aspect of physical mechanism, given that the cancer cells have been observed to migrate rapidly along aligned collagen fibers, it has been assumed that collagen realignment could eliminate the physical barrier impeding the moving cells ${ }^{16}$ and generate tubelike trails of least resistance to promote cell movement ${ }^{14,34}$.
For example, Riching et $\mathrm{al}^{34}$. found that by performing a microchannel migration assay to track the tumor cells traveling in a $3 \mathrm{D}$ collagen gel, the tumor cells were able to travel greater distances in aligned collagen fiber gels compared to the randomly organized collagen gels within the same time period. This observation suggests that the efficiency of tumor migration is enhanced in aligned fibers, possibly by limiting protrusions along the fibers. Some researchers ${ }^{38,39}$ also identified molecular involvement in the facilitated tumor cell movement along the aligned collagen fibers. For example, Wang et al. ${ }^{38}$ found that the cancer cells on highly aligned ECM possessed organized Focal Adhesions (FAs). Highly aligned FAs and associated F-actin stress fibers in turn resulted in the localization of active Rac1 which further stabilized the cell protrusions along the direction of ECM alignment.

The CFOD-TS feature calculated from a small FOV was found to be more discriminating between patients with long and short DFS compared to the features derived from a larger FOV. A possible explanation for this is that the fibers in a small FOV are more likely to constitute a micro-environment, functioning as a unit. The fibers mutually and directly interact with the tumor, and further facilitate tumor migration through the alignment in the orientations, which in turn is quantified as low CFOD-TS. However, a large FOV (big tumor neighborhood) could actually comprise multiple independent fiber-composed micro-environments. In this case, the calculated CFOD-TS not only reflects the fiber orientation alignment within each individual micro-environment as in the case of a small FOV, but also captures the relative orientation alignment among the multiple micro-environments. However, 
(a)

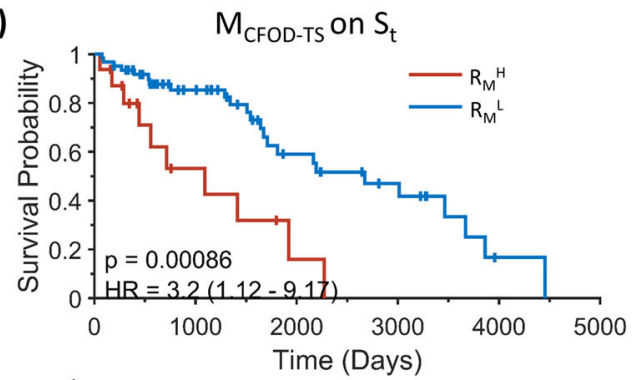

(b)

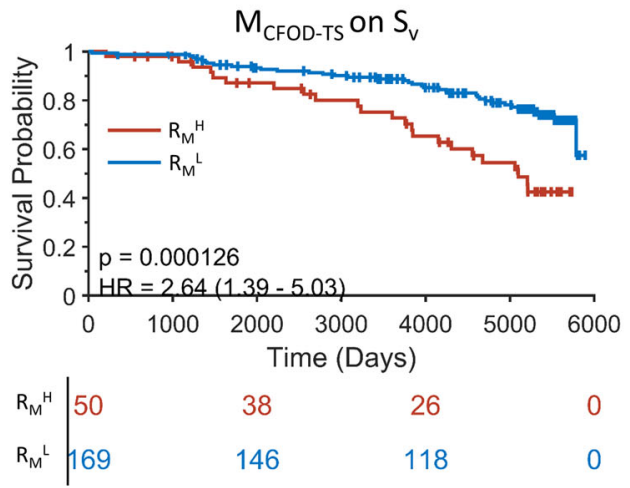

(c)

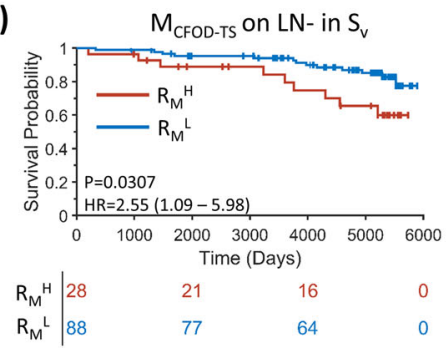

(d)

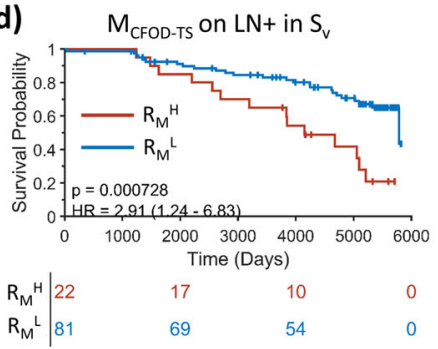

(e)

e) $\mathrm{M}_{\text {CFOD-TS }}$ on intermediate Odx in $\mathrm{S}_{\mathrm{v}}$

Fig. 3 Prognostic performance of $\mathbf{M}_{\text {CFOD-Ts }}$ on $\mathbf{S}_{\mathbf{t}}$ and $\mathbf{S}_{v}$. KM curve estimates of DFS on $R_{M}{ }^{L}$ versus $R_{M}{ }^{H}$ on $S_{t}(\mathbf{a}), S_{v}(\mathbf{b})$ and subgroups in $S_{v}$ (c-e) including subgroup of lymph node negative (c), lymph node positive (d) and intermediate Odx risk category (e) patients. The number of patients at risk at each time point is specified below the KM curves.

considering that the multiple micro-environments within a larger FOV affect the tumor behavior independently, the corresponding calculated CFOD-TS from the large FOV might end up being less prognostic. Additional experiments were also performed to allow for more intuition with respect to the extracted CFOD-TS features. A high correlation between CFOD-TS at the tumor leading edge and across the whole tumor region (Supplementary Fig. 2) indicated the realignment of collagen fibers occurs simultaneously across the entire tumor and is not significantly affected by the spatial location of the fibers. A higher FOV yielded a higher averaged degree and a lower variation in collagen fiber orientation disorganization across the whole tumor region (Supplementary Fig. 3). Considering that the calculation of the disorganization feature within a large FOV takes into account fibers from across a large tumor neighborhood, the results could indicate that fiber orientations were less likely to uniformly align at a large scale (across a large tumor neighborhood). Instead, the orientation alignment was often limited to only those fibers that were adjacently located within the same small tumor neighborhood.

A number of studies have been conducted to interrogate the prognostic value of computerized tumor morphometric features on $\mathrm{H} \& \mathrm{E}$ slide images for IBC, either based on quantitative handcraft histomorphometric features ${ }^{21,23}$ or using deep learning based neural networks ${ }^{40}$. The handcraft morphological attributes have mostly been focused on nuclear histomorphometric features in tumor epithelium or tumor stroma. For example, on a cohort of 378 early stage IBC patients from the same clinical trial (ECOG 2197) as in our study, Verma et $\mathrm{al}^{41}$. built a prognostic model to classify patients into low- versus high-risk groups for recurrence by leveraging the nuclear morphometric signatures from $\mathrm{H} \& \mathrm{E}$ slide images, with $\mathrm{HR}=2.41(95 \% \mathrm{Cl}=1.21-4.79, p=0.01)$. In addition, they found that by combining the nuclear-morphology-based prognostic model with the Odx risk category, 20\% more patients in low risk could be correctly identified compared to using Odx test alone. Similarly, Lu et al. ${ }^{21}$ found that nuclear texture heterogeneity as well as the nuclear orientation disorder were associated with overall survival for early stage IBC patients. Apart from the handcraft features, deep learning based neural network models have also been used to directly prognosticate cancer outcome based on pathology slide images. Wulczyn et al. ${ }^{40}$ developed and trained a deep learning system consisting of a weakly supervised approach and survival loss function using $\mathrm{H} \& \mathrm{E}$ slide images in TCGA. The risk scores generated from the deep learning classifier were validated as being significantly associated with DFS in IBC, after adjusting for the impact of age and stage. However, different from previous studies in computerized histomorphology analysis, our study quantitatively measured collagen fiber orientation organization on H\&E slide images and associated with the breast cancer outcome. Our study demonstrated that CFOD-TS, which is not typically examined to assess disease prognosis in pathological practice, contains potential valuable prognostic information that could be captured by visual inspection on H\&E slides.

In this work, we also explored the molecular underpinning of CFOD-TS on TCGA BRCA dataset with available mRNA sequencing data. Two biological pathways including Cell Cycle Arrest (CCA) and Regulation of Cell Cycle (RCC) were identified as being significantly associated with the $\mathrm{M}_{\mathrm{CFOD}-\mathrm{TS}}$ derived risk scores. $\mathrm{CCA}^{30}$ is often used by cells to facilitate DNA repair and cell cycle progression. A down-regulation of CCA pathway was found in the high-risk patients identified by $\mathrm{M}_{\text {CFOD-TS, which indicated a }}$ dysregulated tumor division process. Similarly, RCC ${ }^{31}$ was also found to be down-regulated among the $M_{C F O D-T S}$ identified highrisk patients, which further suggested a defected regulation of division or duplication process of cancer cells. The mechanism of ECM interacting with the molecular processes within tumor cells has been demonstrated in previous studies ${ }^{42,43}$. Mouw et al. found that the increased ECM stiffness led to reduced expression of the tumor suppressor phosphatase ${ }^{43}$; meanwhile, the increase in ECM stiffness was also identified as one of the causes of collagen fiber realignment ${ }^{42}$. Those findings provide an insight into the possible molecular mechanism behind the association between the 
Table 2. Univariate and Multivariate Cox proportional hazards analysis for DFS on $R_{M} /$ clinicopathological variables/Odx risk category on $S_{t}$ and $S_{t}$.

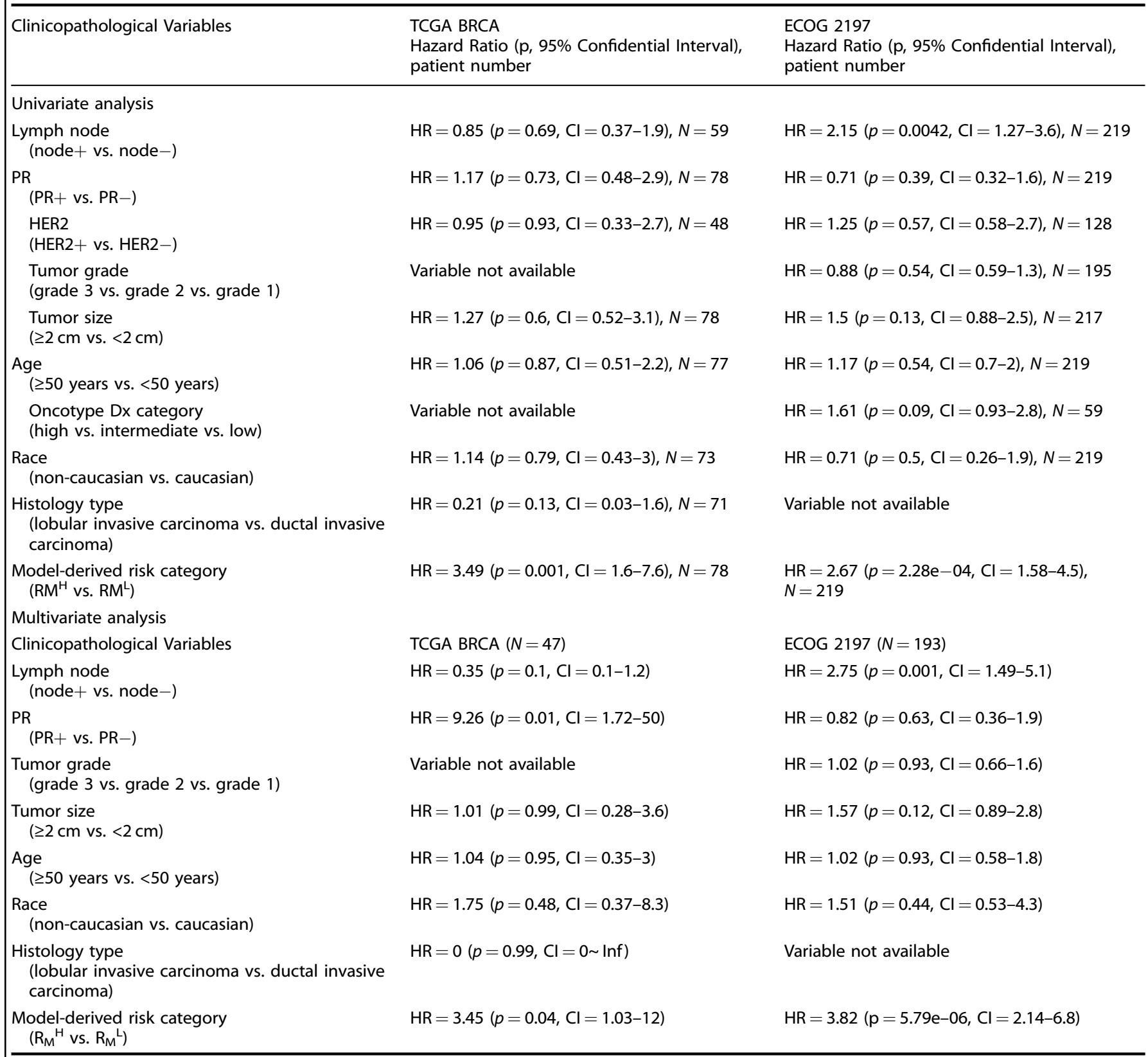

The latter subgroup for each variable (e.g. node -) was used as the reference category for HR calculation

suppressed cell cycle regulation and model-derived collagen orientation risk score developed in our study.

We do acknowledge that the study did have its limitations. Since we did not have access to specialized collagen-specific stained slide images, we were unable to definitively validate the segmentation performance of our algorithm for identifying collagen fibers on the H\&E images. However, we were still able to accurately and quantitatively measure the collagen fiber orientation disorder by capturing the directional pattern of the collagen fibers on H\&E slide images (accuracy was validated by visual inspection from pathologists) without requiring stringent discernment on each individual collagen fiber. Another limitation pertaining to this study is the relatively small data size due to the restrictive patient inclusion criterion to retain the homogenous nature of the cohorts. The purpose of narrowing the scope down to relative homogenous datasets in terms of tumor stage is to eliminate the co-effect of the tumor stage on the CFOD-TS aside from the effect from inherent tumor aggressiveness. Also, unlike the Odx test, which was prospectively validated in both prognostic significance and treatment benefit prediction, our study was retrospectively validated on a single clinical trial dataset and only validated in terms of prognostic ability. Future work will entail validating the pathology image-based prognostic pipeline in larger, additional independent pan-stage cohorts, and also in terms of its predictive benefit for adjuvant chemotherapy. Additionally, we will expand our validation into the pathology images scanned under different parameters (e.g. microns-perpixel) and for other molecular subtypes, such as triple negative breast cancer. Furthermore, we will look to expand our CFOD-TS analysis into tumor adjacent non-oncologic tissue in future 
studies. Specifically, we will use the non-oncologic tissue as a reference to investigate the disruption of CFOD-TS by the tumor cells, and study how the disruption in the organization is associated with the tumor aggressiveness.

In summary, this study quantitatively measured the collagen fiber organization in breast tumor-associated stroma solely using routine $\mathrm{H} \& \mathrm{E}$ stained images and demonstrated its prognostic significance in terms of DFS for early stage ER+ IBC.

\section{METHODS}

\section{Dataset}

Two cohorts were used in this study: TCGA BRCA $\left(\mathrm{S}_{\mathrm{t}}: N=78\right)$ and ECOG $2197\left(S_{\mathrm{v}}: N=219\right)$. A flow diagram describing the inclusion and exclusion criterions on each of the datasets is provided in Supplementary Fig. 1). The primary inclusion criteria for this study were $\mathrm{ER}+$, early stage $(\mathrm{LN}+/ \mathrm{high}$ risk $\mathrm{LN}-$ ) breast cancers, with associated DFS and digital slide presenting a reasonable amount of IBC tissue for subsequent image analysis.

$\mathrm{S}_{\mathrm{t}}$ was employed as a training set for feature discovery and model construction. The dataset included 78 patients with the digital slides of Formalin-Fixed Paraffin-Embedded (FFPE) IBC tissue ( $\times 40$ magnification (mag): $\sim 0.25 \mu \mathrm{m}$ (um)/pixel). The corresponding clinicopathological and outcome data were downloaded from Genomic Data Commons (GDC) data portal. In order to keep relative consistency with respect to tumor stage with $S_{v}$, only the patients with Stage II (Tumor, Node, Metastasis staging system) or high risk (tumor size $>=1 \mathrm{~cm}$ ) $\mathrm{LN}$ - tumors were recruited into this cohort. All the patients with an DFS event (recurrence or death) meeting the inclusion criteria, matched with a set of censored patients (no DFS event) from TCGA BRCA were used to constitute $S_{t}$.

$S_{y}$ was used as an independent validation set to evaluate the model performance. The ECOG 2197 trial was a prospective, randomized, clinical trial from 1998 to 2007 that recruited patients with IBC (1-3 positive LN/LN - with tumor size $>=1 \mathrm{~cm})$ to compare the patient's outcome under two different chemotherapy regimens ${ }^{29} .219$ patients with $E R+I B C$ were selected to comprise $S_{V}$ after the inclusion criteria applied on the 256 patients in ECOG 2197, for whom we had access to both the corresponding de-identified slide images and relevant clinical information. The access to the ECOG dataset involved 2-year process including a proposal review first through ECOG and subsequently through Cancer Therapy Evaluation Program (CTEP) in National Cancer Institute $(\mathrm{NCl})$. In ECOG 2197, the effects of treatment and clinical characteristics of cancers on the outcome were relatively controlled thus enabling the outcome to be more reflective of the nature aggressiveness of breast cancer and making $S_{v}$ an ideal validation set. All the FFPE tissue slides were scanned and digitized using a Philips scanner at 40x mag. Clinicopathological and outcome information were obtained from retrospective chart review.

The study conformed to HIPAA guidelines was approved by the Institutional Review Board (IRB) at University Hospitals Cleveland Medical Center. IRB No 02-13-42C. The need for written consent from participants was waived due to the use of de-identified retrospective data.

\section{Automated detection of collagen fiber orientations in tumor- associated stroma on H\&E slide images}

Following the acquisition of the digital WSIs, HistoQC ${ }^{44}$, a quality control tool for digital pathology slides was employed to identify the useful tumor tissue by excluding fat tissues and regions with artifacts e.g. tissue folding, pen marking, and blurriness.

A conditional Generative Adversarial Network (cGAN) model was implemented on the useful tumor tissues on the digitized WSIs identified by HistoQC. The cGAN model is an extension of GAN with both the generator and discriminator being conditioned on auxiliary information $^{45}$. The cGAN model (deep learning model in Fig. 4b) consisted of a standard U-Net structure as the generator and a multi-layer convolutional network as the discriminator. The model was trained using 1286 images with a pixel size of $512 \times 512$ from a total of $576 \mathrm{H} \& \mathrm{E}$ stained breast cancer pathological images provided by the Netherlands Cancer Institute ${ }^{46}$ and Vancouver General Hospital ${ }^{47}$. All images had annotations of epithelial and stromal regions provided by pathologists under $20 \mathrm{x}$ mag. Following acquisition of cGAN generated epithelial and stroma mask of the breast cancer tissues on WSIs, a set of morphological operations such as image dilation and holes-filling were employed to merge the individual epithelium patches thus automatically generating a tumor mask (Fig. 4b). The stroma region inside the merged tumor mask was defined as tumor-associated stroma. The tumor masks were overlaid on the original tissue images and visually inspected by a pathologist, and, if deemed necessary, manually edited to ensure that the tumor region was accurately delineated.

Collagen fibers are composed of fibril bundles that are linear arrays of type I collagen molecules ${ }^{48}$, thus usually presenting with a linear phenotype on H\&E slide images of breast cancer. A derivative-ofGaussian (DtG) based model described in Griffin et al.'s ${ }^{49}$ work was used to capture the fiber orientation by detecting the linear structures in tumorassociated stroma on H\&E slide images. Specifically, the DtG based model classified each pixel on the slide image as being within one of seven image structures (Flat, Slope, dark Blob, light Blob, dark Line, light Line and Saddle). By retrieving the image pixels identified as belonging to a linear fiber (dark Line structure), we were able to capture the directional pattern of the collagen fibers (Fig. 4e). The accuracy of the collagen fiber orientation detection was visually assessed by an attending pathologist and a resident pathologist independently on 100 tumor tiles randomly cropped respectively from 100 sample WSIs. The tumor tiles were marked with arrows pointing to the fiber orientations captured by our approach. The two pathologists checked the tiles and assigned it to one of four categories (excellent, good, fair and poor) to describe the accuracy of the captured collagen fiber orientations. $94 \%$ of tiles were ranked as excellent/ good, $6 \%$ as fair/poor by the resident pathologist. The attending pathologist ranked $77 \%$ of the tiles as excellent/good, $22 \%$ as fair and $1 \%$ as poor.

\section{Extraction of collagen fiber orientation disorder in tumor- associated Stroma (CFOD-TS) features}

We partitioned the WSI into an array of local tumor neighborhoods where the respective disorder degree of fiber orientations was calculated. Specifically within each individual tumor neighborhood, the direction of each detected linear structure $\left(V_{i}\right)$ representative of collagen fiber orientation was denoted as $\Theta\left(V_{i}\right)$. The $\Theta\left(V_{i}\right)$ was the angle between the horizontal axis and the major axis of a ellipse that has the same secondmoments as $V_{i}$, ranging between 0 degree and 180 degree. The orientations were then discretized to angular bins from 0 to 17. An orientation co-occurrence matrix (Fig. 4f) was constructed based on the set of $\Theta\left(V_{i}\right)$ within the tumor neighborhood (Fig. 4c), with each row/column corresponding to an angular bin. The value for each cell in the matrix was calculated based on the co-occurrence frequency of the two orientations corresponding to the row and column. The equation describing how the orientation co-occurrence matrix was calculated is included in Supplementary Formula 1.

The quantitative measurement of Collagen Fiber Orientation Disorder in Tumor associated Stroma (CFOD-TS) was subsequently calculated from the orientation co-occurrence matrix based on entropy theory ${ }^{28}$ (Fig. 4g). The entropy measurement reflects the level of "information" or "uncertainty" in the collagen fiber's orientation in the neighborhood of the tumor. The equation for orientation disorder calculation is in Supplementary Formula 2. Based on the tiles ranked as fair/poor by the pathologists, the collagen fiber orientation detection algorithm yielded a sub-optimal performance when the tile was dominated by epithelium with a small portion of stroma present as well as when the linear phenotype of the collagen fiber was not present in the tumor-associated stroma of the tile. In order to address these issues, if the stroma ratio (ratio of stromal area to tumor neighborhood) or the number of detected fiber orientations for a tumor neighborhood was lower than a pre-defined threshold, the neighborhood was considered as containing too sparse fiber-enriched stroma content to derive an effective CFOD-TS measurement and thus was disregarded for the subsequent disorganization calculation. Following the calculation of CFOD-TS for each individual compartmentalized tumor neighborhood, an average value of the features derived from all the tumor neighborhoods was calculated as the patient-level feature. The size of tumor neighborhood (FOV) needs to be defined, but no prior knowledge exists as to what the optimal FOV is for collagen fiber disorganization measurement. Therefore, we applied nine different FOVs ranging from 50 to 250 um for feature extraction. Apart from the measurements from the compartmentalized local tumor neighborhoods across the whole tumor region in the WSI, we also assessed the CFOD-TS exclusively in the tumor neighborhoods at the tumor leading edge (within $500 \mu \mathrm{m}$ of the tumor mask periphery), which is roughly the border of tumor cells aggressing and migrating into adjacent normal stromal region. Consequently, we finally obtained a total of 18 features (Supplementary Table 1). 

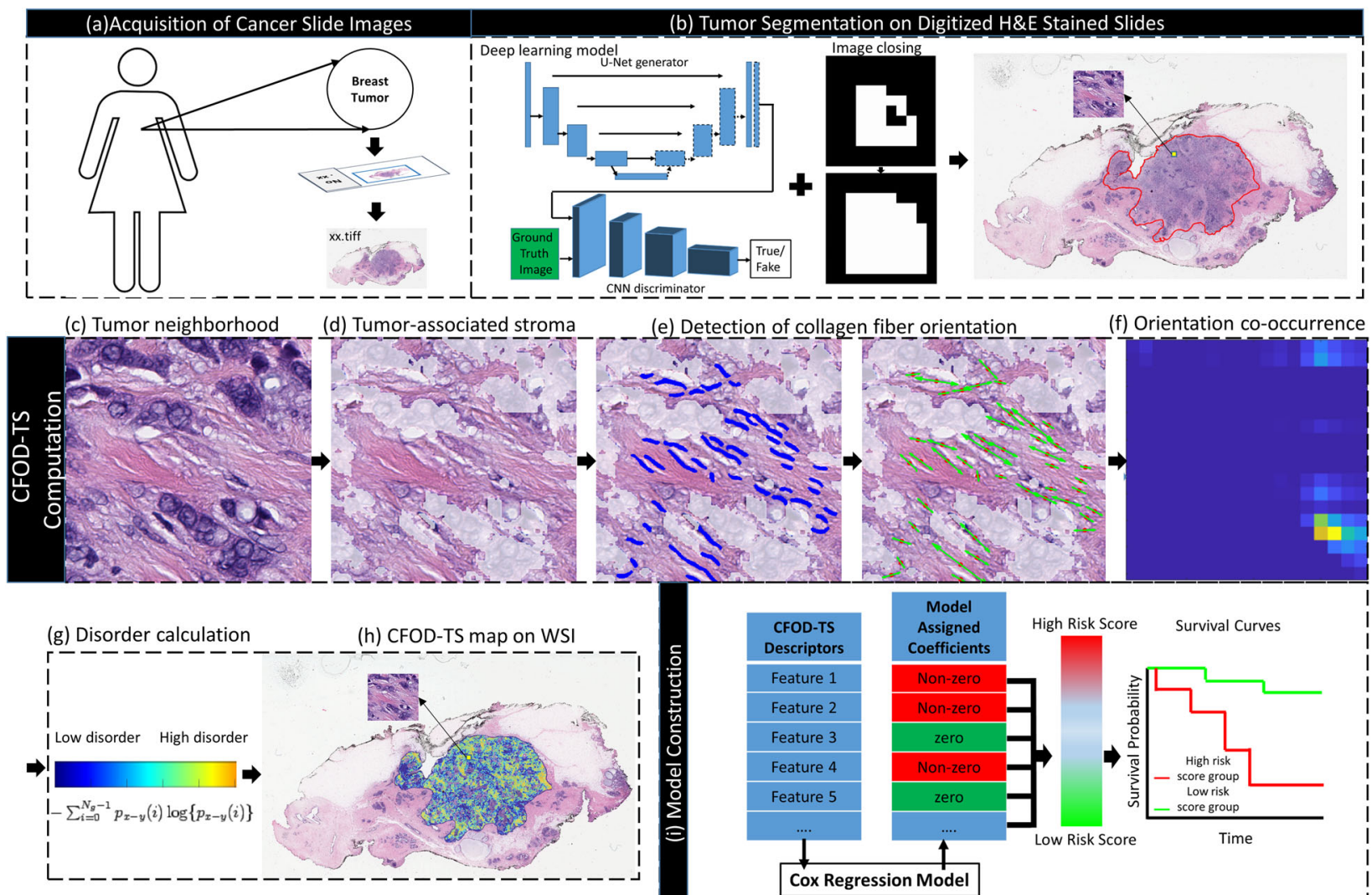

(e) Detection of collagen fiber orientation

(f) Orientation co-occurrence

e) Detection of collagen
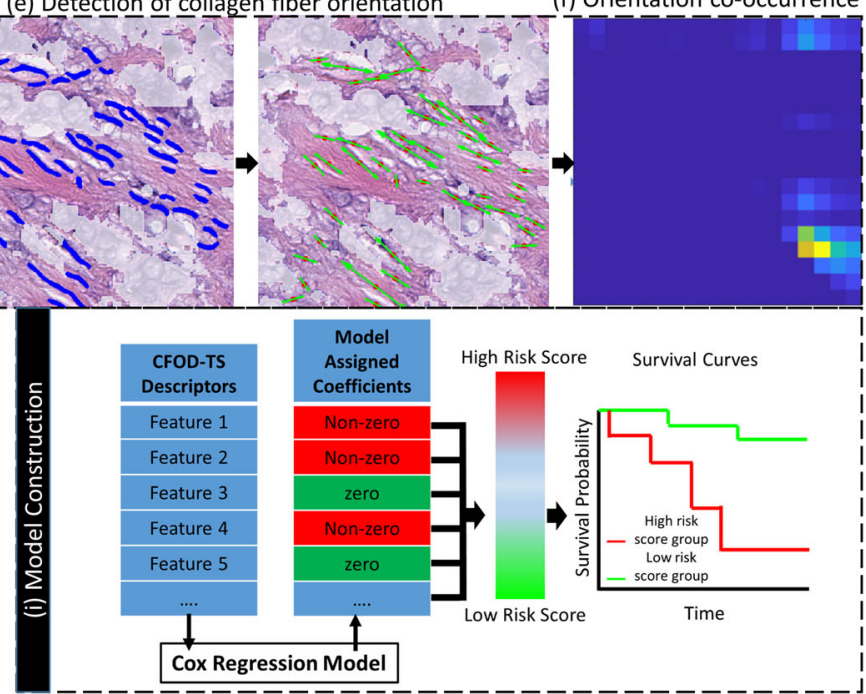

I

Fig. 4 Flowchart of experimental design. The flowchart illustrates the complete experimental design. a Digitized WSIs were collected from two cohorts, in which TCGA BRCA was the training set $\left(\mathrm{S}_{t}\right)$, and ECOG 2197 was the independent validation set $\left(\mathrm{S}_{v}\right)$. b A deep learning model combined with post image processing techniques were utilized to segment the tumor region on WSI. c-h Quantitative features of Collagen Fiber Orientation Disorder in Tumor-associated Stroma were calculated. A tumor neighborhood in a specified radius was defined (c). A deep learning model was applied to the tumor neighborhood to segment the stroma area, which was defined as tumor-associated stroma (d). The collagen fiber orientations in tumor-associated stroma were captured using a linear structure detection-based model (e). An orientation cooccurrence matrix was subsequently constructed with a brighter co-occurrence value in the on-diagonal cells suggesting higher cooccurrence of collagen fibers of the same orientation (f). The feature quantifying the degree of disorder of collagen fiber orientations was then calculated from the matrix (g). The tumor neighborhood slid across the whole tumor region to generate a CFOD-TS feature map (h). The average value of the feature map was calculated at the tumor leading edge as well as across the whole tumor. $i$ Following extraction of a set of CFOD-TS features, A Cox regression model (M $M_{\text {CFOD-TS }}$ ) was constructed on $S_{t}$ to discover top features by assigning a corresponding coefficient to each of the features, based on which a continuous risk score was generated. Kaplan-Meier (KM) survival analysis was performed on the patients with $M_{C F O D-T S}$ derived low risk scores versus high risk scores on both $S_{t}$ and $S_{v}$.

\section{Statistical analysis}

DFS was defined as the time interval between the date of diagnosis/ randomization to the date of recurrence, death and was censored at the date of last follow-up for those patients alive without recurrence. Cox Proportional Hazard Model ${ }^{50}$, henceforth referred to as Cox regression model, relates the time that passes until some event occurs, to multiple covariates. We regularized the Cox regression model by a Least Absolute Shrinkage and Selection Operator (LASSO) penalty function to identify important predictors of DFS. We constructed a Cox regression model named $\mathrm{M}_{\text {CFOD-TS }}$ on $\mathrm{S}_{t}$ between CFOD-TS features and DFS. A corresponding coefficient was assigned to each of the features in the final model. A continuous risk score was then calculated from a linear combination of the top features weighted by the corresponding coefficients for each individual patient (Fig. 4d). The model was further validated on $S_{v}$ by applying the same set of feature coefficients.

Kaplan-Meier (KM) survival analysis was used to analyze the difference of the expected duration of time until an event happens between two defined categories. We converted the continuous risk scores calculated from $M_{C F O D-T S}$ into a binary high $\left(R_{M}{ }^{H}\right.$ : risk score $>$ threshold) vs. low $\left(R_{M}{ }^{L}\right.$ : risk score $<=$ threshold) risk categories. The threshold was selected so as to give the most significant prognosis on $\mathrm{S}_{\mathrm{t}}$. $\mathrm{KM}$ curves were generated to compare the DFS between $R_{M}{ }^{H}$ and $R_{M}{ }^{L}$ groups, with the difference assessed by the log-rank test on both $S_{t}$ and $S_{v}$. Additionally, we performed a dedicated survival analysis for different subgroups in $S_{v}$. KM curves were plotted respectively for the subgroup of $L N+(N=103)$ and $L N-(N=116)$ patients. For the set of patients $(N=59)$ with available Odx scores, we applied the Trial Assigning Individualized Options for Treatment (Rx) (TAILORx) trial recurrence score categorization ${ }^{33}$ (low: $<11$, intermediate: $11-25$, high: $>25$ ) to assign the patients into the low (low recurrence risk), intermediate (ambiguous recurrence risk) and high (high recurrence risk) Odx risk categories. The KM curves were generated for the patients with intermediate Odx category to evaluate whether $\mathrm{M}_{\mathrm{CFOD}-\mathrm{TS}}$ could provide significant prognostic value for the set of patients whose recurrence risk could not be unequivocally interpreted by Odx.

Univariate Cox analysis was performed on clinicopathological variables and Odx risk category to evaluate if any of those clinical routinely examined parameters or the widely used gene test was prognostic of DFS on $\mathrm{S}_{\mathrm{t}}$ or $\mathrm{S}_{\mathrm{v}}$. Additionally, we used multivariate Cox regression analysis to evaluate the independent prognostic significance of $M_{C F O D-T S}$ by adjusting the impact of the clinicopathological variables on DFS. Multivariate analysis was performed on the clinicopathological variables which were available for more than two thirds of patients in each cohort $\left(\mathrm{S}_{t}\right.$ or $\left.\mathrm{S}_{\mathrm{v}}\right)$.

\section{Enrichment analysis of biological pathways}

Histogenomics analysis was performed by investigating the association between biological pathways and CFOD-TS utilizing the normalized Messenger Ribonucleic acid (mRNA) expression data obtained from GDC data portal on TCGA BRCA $\left(S_{t}\right)$. Histogenomics analysis was, however, not conducted on ECOG $2197\left(\mathrm{~S}_{\mathrm{v}}\right)$ due to lack of corresponding genomic data. Wilcoxon Rank Sum Test (WRST) was first used to identify the genes 
significantly differentially expressed between $R_{M}{ }^{H}$ and $R_{M}{ }^{L}$ groups. The identified gene set was in turn taken as the input of Gene Ontology $(\mathrm{GO})^{51,52}$ to identify the associated GO molecular pathways whose member genes were overrepresented in this gene set. After reviewing the list of the identified GO pathways, we selected the ones potentially representative of biological processes underpinning the collagen fiber organization in tumor-associated stroma. The single-sample Gene Set Enrichment Analysis $(\text { ssGSEA })^{53}$ was then used to generate an enrichment score for each of the molecular pathways, assessing the activity level of the pathway in which the member genes were coordinately up- or down-regulated. Unlike GSEA working at the level of sample population, sSGSEA was able to calculate a separate enrichment score for each individual patient. The Pearson correlation was then used to assess the correlation between the enrichment score and the $\mathrm{M}_{\mathrm{CFOD}-\mathrm{TS}}$ derived risk score.

\section{Reporting summary}

Further information on research design is available in the Nature Research Reporting Summary linked to this article.

\section{DATA AVAILABILITY}

The data generated and analyzed during this study are described in the following data record: https://doi.org/10.6084/m9.figshare.14755374 ${ }^{54}$. The majority of the data underlying the claims of this article are openly available in the ten files included in the data record. The remaining data are in the following two files: 'pred risk_label_survival_data(Sv).xlsx', 'clincopathological_survival_data(Sv).xlsx'. These two files are housed on institutional storage and are not publicly available in order to protect patient privacy as informed consent to share participant-level data was not obtained prior to or during data collection. Requests for access to these data should be directed to the corresponding author.

\section{CODE AVAILABILITY}

The code developed and utilized during the current study are available from the corresponding author on reasonable request.

Received: 13 December 2020; Accepted: 25 June 2021; Published online: 06 August 2021

\section{REFERENCES}

1. American Cancer Society. Breast Cancer Facts \& Figures 2019-2020 (American Cancer Society, 2019)

2. Early Breast Cancer Trialists' Collaborative Group (EBCTCG). Effects of chemotherapy and hormonal therapy for early breast cancer on recurrence and 15year survival: an overview of the randomised trials. Lancet 365, 1687-1717 (2005).

3. Davies, C. et al. Relevance of breast cancer hormone receptors and other factors to the efficacy of adjuvant tamoxifen: patient-level meta-analysis of randomised trials. Lancet (London, England) 378 https://pubmed.ncbi.nlm.nih.gov/21802721/ (2011).

4. Paik, S. et al. A multigene assay to predict recurrence of tamoxifen-treated, nodenegative breast cancer. New Engl. J. Med. 351, 2817-2826 (2004).

5. Sparano, J. A. et al. Prospective validation of a 21-gene expression assay in breast cancer. New Engl. J. Med. 373, 2005-2014 (2015)

6. Jaafar, H., Sharif, S. E. T. \& Murtey, M. D. Pattern of collagen fibers and localization of matrix metalloproteinase 2 and 9 during breast cancer invasion. Tumori 100, e204-e211 (2014)

7. Provenzano, P. P. et al. Collagen reorganization at the tumor-stromal interface facilitates local invasion. BMC Med. 4, 38 (2006).

8. Shekhar, M. P., Werdell, J., Santner, S. J., Pauley, R. J. \& Tait, L. Breast stroma plays a dominant regulatory role in breast epithelial growth and differentiation: implications for tumor development and progression. Cancer Res. 61, 1320-1326 (2001).

9. Wang, W. et al. Single cell behavior in metastatic primary mammary tumors correlated with gene expression patterns revealed by molecular profiling. Cancer Res. 62, 6278-6288 (2002).

10. Kolácná, L. et al. Biochemical and biophysical aspects of collagen nanostructure in the extracellular matrix. Physiol. Res. 56, S51-S60 (2007).

11. Conklin, M. W. et al. Aligned collagen is a prognostic signature for survival in human breast carcinoma. Am. J. Pathol. 178, 1221-1232 (2011).

12. Levental, K. R. et al. Matrix crosslinking forces tumor progression by enhancing integrin signaling. Cell 139, 891-906 (2009).
13. Provenzano, P. P. et al. Collagen density promotes mammary tumor initiation and progression. BMC Med. 6, 11 (2008)

14. Egeblad, M., Rasch, M. G. \& Weaver, V. M. Dynamic interplay between the collagen scaffold and tumor evolution. Curr. Opin. Cell Biol. 22, 697-706 (2010).

15. Golaraei, A. et al. Changes of collagen ultrastructure in breast cancer tissue determined by second-harmonic generation double Stokes-Mueller polarimetric microscopy. Biomed. Opt. Express 7, 4054-4068 (2016).

16. Friedl, P. \& Wolf, K. Plasticity of cell migration: a multiscale tuning model. J. Cell Biol. 188, 11-19 (2010)

17. Bredfeldt, J. S. et al. Automated quantification of aligned collagen for human breast carcinoma prognosis. J. Pathol. Informatics 5, 28 (2014).

18. Bredfeldt, J. S. et al. Computational segmentation of collagen fibers from secondharmonic generation images of breast cancer. J. Biomed. Opt. 19, 16007 (2014).

19. Majeed, H., Okoro, C., Kajdacsy-Balla, A., Toussaint, K. C. \& Popescu, G. Quantifying collagen fiber orientation in breast cancer using quantitative phase imaging. $J$. Biomed. Opt. 22, 46004 (2017).

20. Bera, K., Schalper, K. A., Rimm, D. L., Velcheti, V. \& Madabhushi, A. Artificial intelligence in digital pathology - new tools for diagnosis and precision oncology. Nat. Rev. Clin. Oncol. 16, 703-715 (2019).

21. Lu, C. et al. Nuclear shape and orientation features from H\&E images predict survival in early-stage estrogen receptor-positive breast cancers. Lab. Investig. 98 1438-1448 (2018).

22. $\mathrm{Li}, \mathrm{H}$. et al. Quantitative nuclear histomorphometric features are predictive of Oncotype DX risk categories in ductal carcinoma in situ: preliminary findings. Breast Cancer Res. 21, 114 (2019).

23. Whitney, J. et al. Quantitative nuclear histomorphometry predicts oncotype DX risk categories for early stage ER+ breast cancer. BMC Cancer 18, 610 (2018).

24. Lee, G. et al. Cell orientation entropy (COrE): predicting biochemical recurrence from prostate cancer tissue microarrays. Med. Image Comput. Comput. Assist Interv. 16, 396-403 (2013).

25. Wang, X. et al. Prediction of recurrence in early stage non-small cell lung cancer using computer extracted nuclear features from digital H\&E images. Sci. Rep. 7 13543 (2017).

26. Beck, A. H. et al. Systematic analysis of breast cancer morphology uncovers stromal features associated with survival. Sci. Transl. Med. 3, 108ra113-108ra113 (2011).

27. Griffin, J. \& Treanor, D. Digital pathology in clinical use: where are we now and what is holding us back? Histopathology 70, 134-145 (2017).

28. Haralick, R. M. Statistical and structural approaches to texture. Proc. IEEE 67 786-804 (1979)

29. Goldstein, L. J. et al. E2197: Phase III AT (doxorubicin/docetaxel) vs. AC (doxorubicin/cyclophosphamide) in the adjuvant treatment of node positive and high risk node negative breast cancer. JCO 23, 512-512 (2005).

30. cell cycle arrest Gene Ontology Term (GO:0007050). http://www.informatics.jax. org/vocab/gene_ontology/GO:0007050.

31. regulation of cell cycle Gene Ontology Term (GO:0051726). http://www. informatics.jax.org/vocab/gene_ontology/GO:0051726.

32. Corredor, G. et al. Spatial architecture and arrangement of tumor-infiltrating lymphocytes for predicting likelihood of recurrence in early-stage non-small cell lung cancer. Clin. Cancer Res. 2013. https://doi.org/10.1158/1078-0432.CCR-182013 (2018).

33. Sparano, J. A. et al. Adjuvant chemotherapy guided by a 21-gene expression assay in breast cancer. New Engl. J. Med. 379, 111-121 (2018).

34. Riching, K. M. et al. 3D collagen alignment limits protrusions to enhance breast cancer cell persistence. Biophys. J. 107, 2546-2558 (2014).

35. Walsh, A. J., Cook, R. S., Lee, J. H., Arteaga, C. L. \& Skala, M. C. Collagen density and alignment in responsive and resistant trastuzumab-treated breast cancer xenografts. J. Biomed. Opt. 20, 26004 (2015).

36. Seewaldt, V. ECM stiffness paves the way for tumor cells. Nat. Med. 20, 332-333 (2014).

37. Hynes, R. O. The extracellular matrix: not just pretty fibrils. Science 326, 1216-1219 (2009).

38. Wang, W. Y. et al. Extracellular matrix alignment dictates the organization of focal adhesions and directs uniaxial cell migration. APL Bioeng. 2, 046107 (2018).

39. Provenzano, P. P., Inman, D. R., Eliceiri, K. W., Trier, S. M. \& Keely, P. J. Contact guidance mediated three-dimensional cell migration is regulated by Rho/ROCKdependent matrix reorganization. Biophys. J. 95, 5374-5384 (2008).

40. Wulczyn, E. et al. Deep learning-based survival prediction for multiple cancer types using histopathology images. PLoS ONE 15, e0233678 (2020).

41. Verma, N. et al. Image-based risk score to predict recurrence of $E R+$ breast cancer in ECOG-ACRIN Cancer Research Group E2197. J. Clin. Oncol. 36, 540-540 (2018).

42. Pirentis, A. P. et al. Remodelling of extracellular matrix due to solid stress accumulation during tumour growth. Connect Tissue Res. 56, 345-354 (2015).

43. Mouw, J. K. et al. Tissue mechanics modulate microRNA-dependent PTEN expression to regulate malignant progression. Nat. Med. 20, 360-367 (2014). 
44. Janowczyk, A., Zuo, R., Gilmore, H., Feldman, M. \& Madabhushi, A. HistoQC: an open-source quality control tool for digital pathology slides. JCO Clin. Cancer Informatics https://doi.org/10.1200/CCl.18.00157 (2019).

45. Mirza, M. \& Osindero, S. Conditional generative adversarial nets. Preprint at https://arxiv.org/abs/1411.1784 (2014).

46. van 't Veer, L. J. et al. Gene expression profiling predicts clinical outcome of breast cancer. Nature 415, 530-536 (2002).

47. Beck, A. H. et al. Systematic analysis of breast cancer morphology uncovers stromal features associated with survival. Sci. Transl. Med. 3, 108ra113 (2011).

48. Lodish, H. et al. in: Molecular Cell Biology 4th edn (New York: W. H. Freeman, 2000).

49. Griffin, L. D., Lillholm, M., Crosier, M. \& van Sande, J. in: Scale Space and Variational Methods in Computer Vision (eds. Tai, X. C., Mørken, K., Lysaker, M., Lie, K. A.) (Springer, 2009).

50. Cox, D. R. Regression models and life-tables. J. R. Stat. Soc. Ser. B: Methodological 34, 187-220 (1972).

51. The Gene Ontology Consortium. The gene ontology resource: 20 years and still GOing strong. Nucleic Acids Res. 47, D330-D338 (2019).

52. Ashburner, M. et al. Gene Ontology: tool for the unification of biology. Nat. Genet. 25, 25-29 (2000).

53. Barbie, D. A. et al. Systematic RNA interference reveals that oncogenic KRAS -driven cancers require TBK1. Nature 462, 108-112 (2009).

54. Li, H. Metadata record for the article: Collagen Fiber Orientation Disorder from H\&E images is prognostic for early-stage breast cancer: Clinical Trial Validation. figshare https://doi.org/10.6084/m9.figshare.14755374 (2021).

\section{ACKNOWLEDGEMENTS}

Research reported in this publication was supported by the National Cancer Institute under award numbers 1U24CA199374-01, R01CA202752-01A1, R01CA208236-01A1, R01 CA216579-01A1, R01 CA220581-01A1, 1 U01 CA239055-01, 1U01CA248226-01, 1U54CA254566-01, National Heart, Lung and Blood Institute, 1R01HL15127701A1, National Institute for Biomedical Imaging and Bioengineering 1R43EB028736-01, National Center for Research Resources under award number 1 C06 RR12463-01, VA Merit Review Award IBX004121A from the United States Department of Veterans Affairs, Biomedical Laboratory Research and Development Service, the Office of the Assistant Secretary of Defense for Health Affairs, the Breast Cancer Research Program (W81XWH-19-1-0668), the Prostate Cancer Research Program (W81XWH-15-1-0558, W81XWH-20-1-0851), the Lung Cancer Research Program (W81XWH-18-1-0440, W81XWH-20-1-0595), the Peer Reviewed Cancer Research Program (W81XWH-18-10404), the Kidney Precision Medicine Project (KPMP) Glue Grant, the Ohio Third Frontier Technology Validation Fund, the Clinical and Translational Science Collaborative of Cleveland (UL1TR0002548) from the National Center for Advancing Translational Sciences (NCATS) component of the National Institutes of Health and $\mathrm{NIH}$ roadmap for Medical Research, The Wallace H. Coulter Foundation Program in the Department of Biomedical Engineering at Case Western Reserve University. The content is solely the responsibility of the authors and does not necessarily represent the official views of the National Institutes of Health, the U.S. Department of Veterans Affairs, the Department of Defense, or the United States Government.

\section{AUTHOR CONTRIBUTIONS}

H.L., K.B., and A.M. conceptualized and designed the study. H.L., Z.Z., and C.L. were responsible for the experiment run. H.G., L.G., and N.D. were responsible for providing the image data and corresponding clinical information. P.T. was responsible for checking the breast tumor annotations. P.T., A.G., and A.H. were responsible for validating the detection of collagen fiber orientations. H.L., K.B., and P.T. were responsible for the analysis of underlying biological pathways. P.F. supervised the statistical analysis. M.F. and S.G. were responsible for providing the biological interpretation of the findings. The manuscript was written primarily by H.L., K.B., and A.M., with support from all authors. All authors have read and approved the manuscript.

\section{COMPETING INTERESTS}

Dr. Madabhushi is an equity holder in Elucid Bioimaging and in Inspirata Inc. In addition, he has served as a scientific advisory board member for Inspirata Inc, Astrazeneca, Bristol Meyers-Squibb and Merck. Currently he serves on the advisory board of Aiforia Inc. He also has sponsored research agreements with Philips and Bristol Meyers-Squibb. His technology has been licensed to Elucid Bioimaging. He is also involved in a NIH U24 grant with PathCore Inc, and 3 different R01 grants with Inspirata Inc. Dr. Ganesan has consulted for Merck, Roche, Foundation Medicine, Foghorn Therapeutics, Inspirata, Novartis and EQRX. $\mathrm{He}$ is also on the Scientific Advisory Board of Silagene. In addition, he has equity in Silagene and Inspirata and research funding from M2Gen. His spouse is an employee of Merck and has equity in Merck. The remaining authors declare no competing interests.

\section{ADDITIONAL INFORMATION}

Supplementary information The online version contains supplementary material available at https://doi.org/10.1038/s41523-021-00310-z.

Correspondence and requests for materials should be addressed to H.L. or A.M.

Reprints and permission information is available at http://www.nature.com/ reprints

Publisher's note Springer Nature remains neutral with regard to jurisdictional claims in published maps and institutional affiliations.

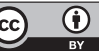

Open Access This article is licensed under a Creative Commons Attribution 4.0 International License, which permits use, sharing, adaptation, distribution and reproduction in any medium or format, as long as you give appropriate credit to the original author(s) and the source, provide a link to the Creative Commons license, and indicate if changes were made. The images or other third party material in this article are included in the article's Creative Commons license, unless indicated otherwise in a credit line to the material. If material is not included in the article's Creative Commons license and your intended use is not permitted by statutory regulation or exceeds the permitted use, you will need to obtain permission directly from the copyright holder. To view a copy of this license, visit http://creativecommons. org/licenses/by/4.0/.

(c) The Author(s) 2021 